\title{
Using Fuzzy Ontology to Improve Similarity Assessment: Method and Evaluation
}

\author{
Hoda Safaeipour, ${ }^{1}$ M. H. Fazel Zarandi, ${ }^{1, *}{ }^{\text {Susan Bastani }}{ }^{2}$
}

\footnotetext{
${ }^{1}$ Department of Industrial Engineering, Amirkabir University of Technology, Tehran 15875-4413, Iran.

${ }^{2}$ Department ofSocial Science, Alzahra University, Tehran, Iran.
}

\begin{abstract}
Assessing semantic similarity is a fundamental requirement for many AI applications. Crisp ontology (CO) is one of the knowledge representation tools that can be used for this purpose. Thanks to the development ofsemantic web,CO-based similarity assessment has become a popular approach in recent years. However, in the presence of vague information, $\mathrm{CO}$ cannot consider uncertainty of relations between concepts. On the other hand, fuzzy ontology (FO) can effectively process uncertainty of concepts and their relations. This paper aims at proposing an approach for assessing concept similarity based on FO. The proposed approach incorporates fuzzy relation composition in combination with an edge counting approach to assess the similarity. Accordingly, proposed measure relies on taxonomical features of an ontology in combination with statistical features of concepts. Furthermore, an evaluation approach for the FO-based similarity measure named as FOSE is proposed. Considering social network data, proposed similarity measure is evaluated using FOSE. The evaluation results prove the dominance of proposed approach over its respective CO-based measure.
\end{abstract}

Keywords: Knowledge representation, Concept similarity, Evaluation approach, Evaluation results, Process uncertainties, Semantic similarity, Similarity assessment, Similarity measure, Statistical features, Ontology. 